\title{
Una mirada al Ordenamiento Territorial en Venezuela en el Marco del Ecosocialismo
}

Rosa Corina García

\section{Resumen}

El presente documento tiene por finalidad hacer referencia al proceso de ordenación del territorio en Venezuela, su vinculación con la nueva visión planteada que confiere el Plan Patria 2013-2019 y los patrones de desarrollo que exige actualmente el país. En tal sentido, primeramente se hace referencia al marco legal que rige la ordenación del territorio en Venezuela, el proceso de ordenación territorial, los instrumentos de ordenación del territorio que se han construidopara su gestión en el extinto Ministerio del Poder Popular para el Ambiente (MINAMB), (hoy Ministerio del Ecosocialismo y Aguas-(MINEA), los ámbitos que abarca la ordenación del territorio, tanto en espacios denominados áreas ordinarias, como en los espacios protegidos; los enfoques metodológicos seguidos en dicho proceso y su relación con los paradigmas del Ecosocialismo y Ecodesarrollo, lo que justifica su divulgación en el marco de los esquemas de ordenación territorial. La investigación es de carácter ontológico, ya que se encuentra subyacente la experiencia habida como funcionaria en las actividades de ordenación territorial a nivel técnico y gerencial en el extinto MINAMB; también es de carácter descriptivo y documental lo que permitió realizar un análisis situacional que condujeron a unas conclusiones sobre la materia presentada, en las cuales se destaca, que la metodología de ordenamiento territorial integral y participativa seguida en el MINAMB, derivó en un espiral de aprendizaje colectivo e implicó un replanteamiento epistemológico, político y metodológico, con instrumentos de planificación elaborados desde una óptica holística con y para la comunidad, en concordancia con el entorno ambiental.

Palabras clave: Ordenación del territorio, Ecosocialismo, Participación Comunitaria, Instrumentos de gestión territorial. 


\section{Abstract}

This document is intended to refer to the process of land use planning in Venezuela, its link with the new raised vision that gives the homeland Plan 2013-2019 and patterns of development that currently requires the country. In this sense, first reference is made to the legal framework governing the management of territory in Venezuela, the spatial planning process, the instruments of regional planning which have been constructed for its management in the extinct Ministry of the Popular power for the environment (MINAMB), (today Ministry of Ecosocialism and waters-(MINEA), areas encompassing the planning), both in areas called areas ordinary, as in those spaces protected; them approaches methodological followed in said process and its relationship with them paradigms of the Ecosocialism and Ecodevelopment, what justifies its disclosure in the frame of the schemes of management territorial. Research is ontological in nature, as is underlying the experience because as an officer in the activities of regional planning to technical and managerial levels in the extinct MINAMB; It is also descriptive and documentary character which allowed to conduct a situational analysis that led to conclusions on the matter presented, in which stands out the methodology of comprehensive land management and participatory followed in MINAMB, led to a spiral of collective learning and involved a rethinking epistemological, political and methodological, with planning tools developed from a holistic optics with and for the community, in concordance with the environment environmental.

Key words: management of the territory, Ecosocialism, community participation, territorial management instruments.

Rosa Corina García. (garciarosa815@gmail.com), Universidad Nacional Experimental de la Fuerza Armada Nacional (UNEFA)-Chuao, Caracas-Venezuela. Programa de Maestría en Gerencia Ambiental 


\section{INTRODUCCIÓN}

El estilo de desarrollo conlleva a un modelo territorial que es la proyección en el espacio geográfico, de las políticas sociales, económicas, ambientales y culturales de una sociedad. El concepto de ordenación del territorio que refiere la Ley Orgánica para la Ordenación del Territorio (LOPOT), (1983), en su artículo № 2 , seguido conceptualmente en el presente documento, está referido a la regulación y promoción de la localización de los asentamientos humanos, actividades económicas y sociales de la población, y el desarrollo físico espacial para lograr una armonía entre el mayor bienestar de las comunidades, la organización y uso de los recursos naturales y la protección y valorización del ambiente, enfocado desde el punto de vista del desarrollo integral.

Es una política de Estado, que se articula a instrumentos de orden administrativo, legal y político institucional. Siendo un proceso planificado, se vincula a varias etapas de planificación; su naturaleza técnica y sociopolítica requiere del manejo de fundamentos teóricos, procedimientos y técnicas y concierne a toda la sociedad. Posee asimismo un carácter económico, pues interviene en la estructura de la base productiva asignando usos al territorio y orientando la localización de las actividades; tiene el apoyo de una base financiera, y evidentemente, posee una naturaleza cultural que responde al conjunto de valores que conforman la base de la cultura nacional que expresa las aspiraciones de las comunidades.

Dado el mandato legal y la importancia del ordenamiento territorial, se presenta este documento, referido al proceso de ordenación del territorio en Venezuela y su vinculación con la visión de país, conferida mediante el Plan Patria 20132019. Para ello se considera el marco jurídico y ambiental que rige la ordenación territorial en Venezuela, las metodologías aplicadas recientemente por el Ministerio del Poder Popular Para el Ambiente (MINAMB) como autoridad ambiental, y su relación con nuevos paradigmas de desarrollo, tales como el Ecosocialismo y el Ecodesarrollo,lo que justifica su divulgación en el marco de nuevas estrategias de planificación territorial; todo ello, desde una mirada ontológica, así como documental y descriptiva, para finalmente presentar las conclusiones derivadas del proceso de análisis realizado. 


\section{OBJETIVOS}

\section{Objetivo General}

Evaluar el proceso de ordenación del territorio en Venezuela y su vinculación con la visión planteadaen el Plan Patria 2013-2019, en el marco del Ecosocialismo.

\section{Objetivos Específicos}

- Describir el proceso de ordenación del territorio en Venezuela en función del marco legal ambiental.

- Evaluar el proceso de ordenación del territorio en Venezuela, en función de visión planteada en el Plan Patria 2013-2019.

- Considerar las metodologías para la ordenación del territorio utilizadas en la elaboración de los instrumentos de planificación y gestión territorial.

\section{Marco jurídico ambiental que rige la ordenación del territorio en Venezuela}

La planificación del ordenamiento territorial de Venezuela está fundamentado en el marco legal del país, el cual parte de la Constitución de la República Bolivariana de Venezuela (CRBV), la cual establece en sus artículos 120, 127 y 128,del aprovechamiento de los recursos naturales, protección del ambiente y procesos ecológicos, y en especial, la política de ordenación del territorio atendiendo a realidades ecológicas, geográficas, poblacionales, sociales, culturales, económicas y políticas, de acuerdo al desarrollo sustentable.

El marco jurídico de la planificación territorial en Venezuela, ha tomado en cuenta aspectos ambientales, demográficos y peculiaridades en cuanto a los bienes naturales presentes, y se han elaborando un conjunto de instrumentos de planificación que obedecen al mandato legal, concebidos de forma jerarquizada y fundamentados en la Ley Orgánica Para la Ordenación del Territorio LOPOT (1983),donde están contenidas las disposiciones que rigen el proceso de ordenación del territorio en concordancia con la Estrategia de Desarrollo Económico y Social a largo plazo de la Nación.

Asimismo, son considerados en el proceso de ordenamiento territorial, instrumentos legales vinculados directamente con la ordenación del territorio, la elaboración de la cartografía, la atención al riesgo y al ambiente, así como aquellos vinculados con la participación ciudadana, entre otros. 
Por otra parte, la Ley del Plan de la Patria 2013-2019, (2013), en su Objetivo Histórico N 5 señala: «Contribuir con la preservación de la vida en el planeta y la salvación de la especie humana», la cual debe realizarse mediante una adecuada ordenación territorial que garantice el buen uso de los bienes naturales para el beneficio del colectivo, asimismo, la Ley Orgánica del Ambiente LOA,(2006) establece las disposiciones y los principios rectores para la gestión del ambiente en el marco del desarrollo sustentable, como derecho y deber fundamental del Estado y de la sociedad, para contribuir a la seguridad y al logro del máximo bienestar de la población y sostenimiento del planeta en interés de la humanidad, y finalmente, el Plan Nacional de Ordenación del Territorio (1998) instrumento de gestión territorial a nivel nacional, del cual se conoce una actualización para el año 2011.

\section{Una mirada a la ordenación territorial en Venezuela}

Los ámbitos de ordenación del territorio en Venezuela, están englobados en las llamadas áreas de régimen ordinario y las áreas protegidas. Las áreas de régimen ordinario la conforman todas aquellas zonas del país que no están bajo figuras jurídicas de protección; mientras que las áreas protegidas gozan de esta condición. En relación a estas áreas de régimen ordinario, el país ha aunado esfuerzos importantes en la elaboración de planes de ordenación para las entidades federales, así como también, planes de manejo para recursos tales como los forestales y aguas.

Respecto a las áreas protegidas, en Venezuela éstas se encuentran reguladas a través de diferentes leyes y reconocidas desde la Constitución de la República Bolivariana de Venezuela (1999). Según la legislación venezolana se denominan Áreas Bajo Régimen de Administración Especial (ABRAE). Su importancia radica en hacer un uso racional de los recursos ambientales, frenar conflictos sociales y detener la degradación de los ecosistemas. En este sentido, se plantea como prioridad proponer un mejor ordenamiento del espacio que abarcan las ABRAE dentro del marco socioeconómico y ecológico, que tome en cuenta las características y distribución de la población, el proceso de análisis y la organización de los ecosistemas en estas áreas protegidas.

En el caso del ordenamiento territorial de otro tipo de $A B R A E$, se han tenido presentes premisas cónsonas con el tipo de figura a ordenar y su objetivo de creación, para la propuesta de zonas de protección y conservación, de esta manera también se relaciona con el art. 12 de la Ley Orgánica de Seguridad de la Nación LOSN (GOE- 
6156-E, 2014). Así, Venezuela tiene un conjunto de ABRAE, tales como parques nacionales, monumentos naturales, reservas de biosfera, entre otras, (ver Figura $N^{0} 1$ ), las cuales coadyuvan con la defensa de la soberanía nacional a través de la protección y conservación de los bienes naturales que albergan. La planificación de la ordenación del territorio, se realiza tomando en consideración las aptitudes de los diferentes espacios para el establecimiento de usos y actividades los cuales se engloban en un sistema de planes de los cuales forman parte el Plan Nacional de Ordenación del Territorio (PNOT); los planes estadales de ordenación del territorio, los planes regionales y los planes de ordenamiento territorial de ABRAE, entre otros.

Debido a que una de las directrices principales del PNOT es la mejor localización de las actividades económicas, implica el establecimiento de normas claras, precisas y estables en lo que se refiere a las posibilidades de uso del territorio nacional, estrategia que también es considerada en los planes estadales de ordenación del territorio, planes urbanos y planes de sitio. Igualmente, para la gestión de las $A B R A E$, los planes de ordenamiento de las mismas, conforman los instrumentos técnicolegales de planificación espacial fundamentales para su conservación o protección, y donde se establecen las directrices para la gestión, de estas áreas, en armonía con los tres grandes objetivos generales del desarrollo integral, con especial énfasis a la participación ciudadana, activa, protagónica y corresponsable, donde el trabajo a realizar, sea una responsabilidad compartida entre los ciudadanos y el Estado. 


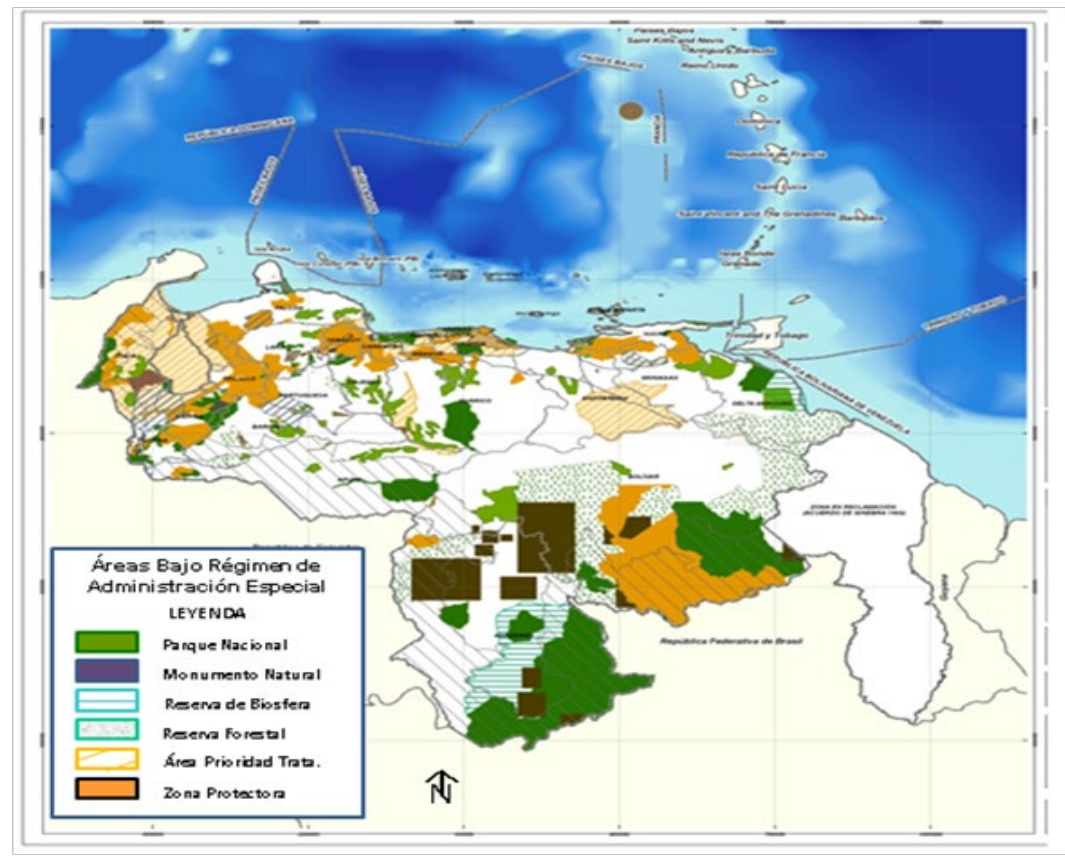

Figura 1. Áreas Bajo Régimen de Administración Especial en Venezuela Fuente: (MINAMB, 2011)

\section{Procedimientos metodológicos aplicados en la ordenación territorial}

Existen diversos planteamientos metodológicos para el abordaje de la elaboración de proyectos de ordenamiento territorial con planificación participativa, pero es común a ellos, la construcción conjunta del producto, mediante procesos dinámicos entre la relación de la comunidad y las instituciones; de esta manera se establece una comunicación bidireccional, dinámica y constante en la búsqueda del enriquecimiento de saberes, y la elaboración de instrumentos de planificación producto de la unificación de criterios y con sentido de pertenencia.En Venezuela, dentro de estas metodologías utilizadas recientemente por el MINAMB, se puede hacer referencia a la Metodología de Análisis Integral de Ecosistemas (Berroterán, 2004), (ver Figura $N^{0} 2$ ), la cual fue aplicada en el ejercicio del ordenamiento territorial de la Reserva Forestal El Caura y de la Reserva Forestal Imataca, con resultados considerados positivos, por su carácter integrador entre los ámbitos físico natural, con el socioeconómico y cultural producto las relaciones entre las fases que la conforman. 
La experiencia habida en el Ministerio del Poder Popular para el Ambiente (MINAMB) hoy (Ministerio de Ecosocialismo y Aguas-MINEA), en cuanto a la aplicación de este proceso metodológico integrador y autormatizado, ha generado que recientemente se hayan realizado planes de ordenación territorial estadal y de ABRAE, en las cuales se ha vinculado la comunidad en todas sus fases, tales como en los casos de los planes de ordenamiento territorial de las reservas forestales Imataca y Caura, así como a espacios de régimen ordinario: Plan de Ordenación del Territorio del Estado Monagas (POTEM) ydel Plan de Ordenación del Territorio del Estado Bolívar (POTEB), por citar algunos. En estos casos, la comunidad tuvo alta participación, efectuó su propia zonificación (caso del territorio indígena) y colaboró en la zonificación y asignación de usos del resto de las áreas bajo planificación territorial. Asimismo, tradujo a su propia lengua originaria, el contenido del instrumento (Caso Reserva Forestal El Caura del estado Bolívar), lo que permite mayor vinculación y apropiamiento del Plan.

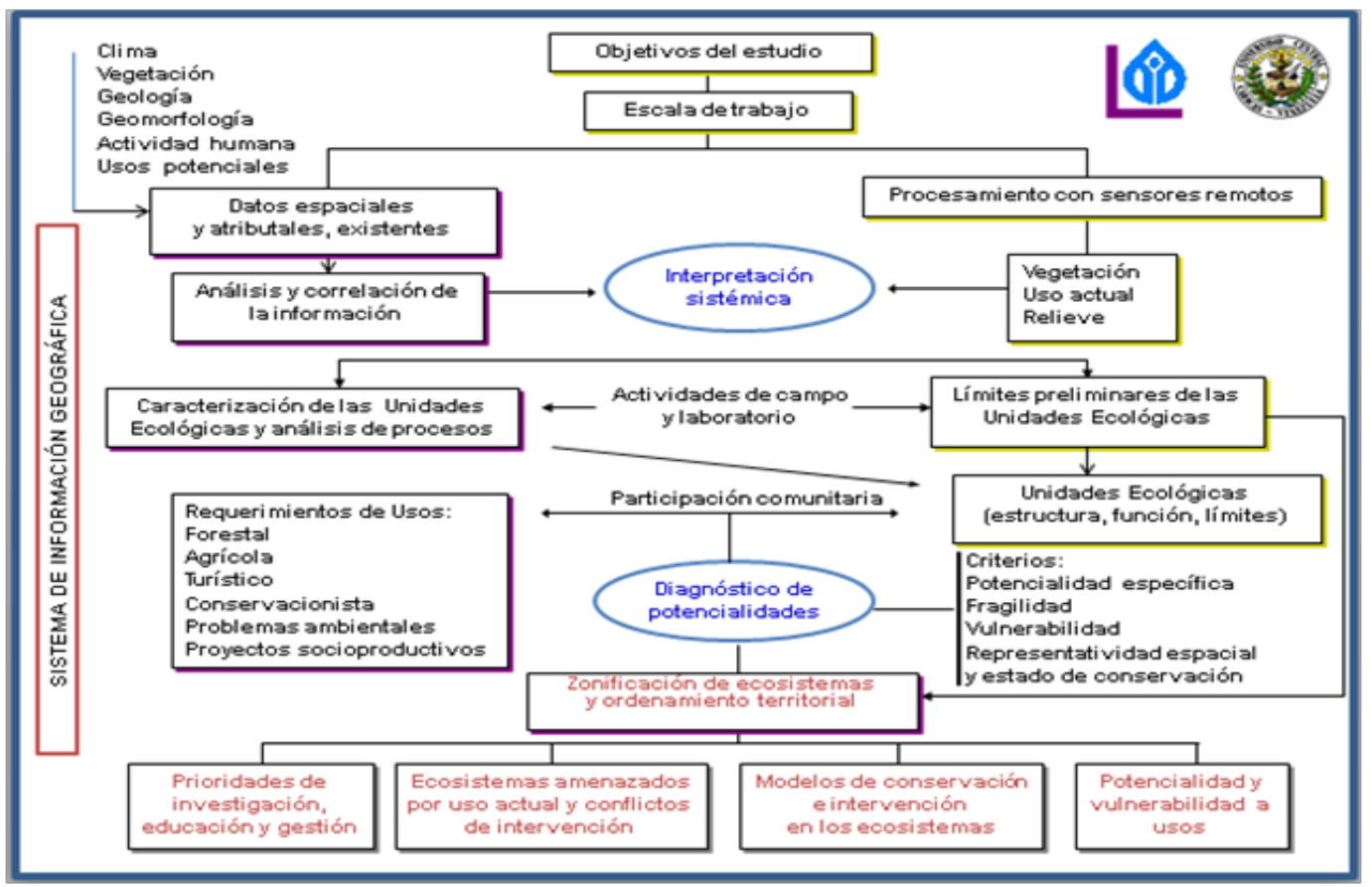

Figura 2. Metodología de Análisis Integral de Ecosistemas. (MINANB, 2008) 
Las fases genéricas que enmarcan la implementación del proceso metodológico que permite elaborar los planes de ordenación, son de acuerdo al MINAMB (2008):

a) Sensibilización a las comunidades con relación al tipo de plan por ejecutar e invitación a participar en el proceso de ordenamiento territorial: esta fase es el primer contacto con las comunidades en función de las actividades que van a realizarse;

b) Inserción de la comunidad en el proceso de planificación,mediante variados mecanismos de participación;

c) Elaboración del diagnóstico participativo. Este se realiza mediante el aporte institucional y comunitario en el proceso de identificación y propuestas de resolución de problemas, de desarrollo socioproductivo y en la directa construcción de los productos;

d) Elaboración del Plan, el cual se construye desde la zonificación territorial, con base a las potencialidades y restricciones del área, y fundamentándose en el marco legal vigente. Asimismo Implica la recopilación de propuestas de usos y de actividades socioproductivas permitidas, los mecanismos de financiamiento y la responsabilidad de acción de cada ente con la comunidad;

e) Revisión del Plan elaborado. Este se realiza en asamblea de ciudadanas y ciudadanos;

f) Consulta Pública del Plan;

g) Implementación del Plan, donde debe especificarse la responsabilidad que cada actor tiene dentro del mismo, y

h) Control, revisión y actualización del Plan, en el tiempo en el cual se especifique. De esta manera la comunidad participa en el la elaboración del documento técnico, en la formulación de la zonificación territorial, en el abordaje de los problemas ambientales y su solución, y en la elaboración del proyecto de decreto que contiene la propuesta de ordenación del territorio.

\section{Resultados}

La mayoría de las entidades federales en Venezuela, cuenta a la fecha, con su respectivo plan de ordenación estadal; sin embargo,algunosse encuentran desactualizados, encontrándose situaciones donde los procesos de ocupación y 
uso del territorio se han producido de manera anárquica, originándose problemas ambientales, que tienen expresión en problemas sociales einclusive de salud.Esto ocurre, porque en ciertos casos, los planes que se han formulado han producido efectos opuestos a los objetivos para los cuales fueron elaborados, González (1983), y se han adoptado técnicas e instrumentos provenientes de otras realidades geográficas, con otro contexto social, económico y administrativo. En tal sentido, la actualidad exige que los planteamientos y objetivos del modelo territorial deban definirse y adoptarse con amplia participación y consenso de todos los actores involucrados. Del mismo modo, la particularización y localización espacial de los problemas ambientales, así como las necesidades más urgentes de los ciudadanos, deben ser materia esencial de la planificación; por ende es menester elaborar dichos planes de ordenación territorial atendiendo no solo a las realidades geográficas, sino al empleo de procesos metodológicos que consideren a las características ecosistémicas de los bienes naturales propios de cada área; construir el plan de ordenación territorial con una metodología integradora, con carácter estratégico y concebido en un proceso vinculado a un programa de acción territorial. Se trata de un instrumento de visión prospectiva, de contenidos específicos y de acciones concretas que se desarrollen a través del tiempo, sujetos a una serie de principios ambientales, económicos y sociales, con la adopción del concepto de flexibilidad como fundamento de las innovaciones propuestas, tal y como refiere (CEPAL, 2001). Su esencia consiste en permitir la adecuación de las disposiciones del Plan a la realidad cambiante, y ser una guía orientadora de las actuaciones públicas y privadas.

\section{Discusión}

El desarrollo, como hasta ahora ha sido concebido hay que reconsiderarlo y convertirlo en un elemento que combine eficazmente la naturaleza y las comunidades, así como también que garantice los bienes naturales. El Ecosocialismo, según (Lowvy, 2004) es considerado como un enfoque de desarrollo emergente, basado en el repensar la relación humanidad-naturaleza, cuya lógica debe construirse desde la comunidad, para la preservación de la vida en el planeta. Esto en Venezuela justifica el Quinto Objetivo Histórico del Plan de la Patria: "Contribuir con la Preservación de la vida en el Planeta y la Salvación de la Especie Humana". Es pues pertinente, de la manera más prospectiva, avanzar en la confección de un modelo de escalamiento productivo de transición,einsistir junto a las comunidades, en darle las debidas coherencias a las políticas de forma más organizada, para avanzar en las distintas dimensiones que encierra el Quinto Objetivo Histórico. 
Ello implica un modelo de desarrollo humanista, que coloque al ciudadano y ciudadana en el centro de su atención, conciliando su relación con el ambiente, impulsando un nuevo modelo de producción y consumo, garantizados mediante un compromiso establecido a través de la participación popular; por tanto, se impone una nueva planificación, ajustada a cada medio, de acuerdo a las aptitudes territoriales presentes expresadas en la zonificación territorial, que permita asumir la pluralidad de actores, los cambios y también admitir tendencias establecidas que deban ser replanteadas por otras nuevas, incorporado un componente de flexibilidad y una visión endógena, lo cual puede ajustarse también al esquema del llamado Ecodesarrollo, adaptado a cada medio, según sus aptitudes territoriales, pues este último alberga ideas que según PNUMA, (1978) lo define así:

El ecodesarrollo es un estilo o modelo para el desarrollo de cada ecosistema, que además de los aspectos económicos, considera de manera particular los datos económicos y culturales del propio ecosistema para optimizar un aprovechamiento, evitando la degradación del medio ambiente y las acciones depredadoras.

En este sentido se traduce en un proceso de transición mediante una planificación que busca la articulación de dos metas: por un lado, la meta de desarrollo, dirigida a mejorar la calidad de vida, mediante el incremento de productividad, y por otro, la de mantener en balance o equilibrio el ecosistema donde se desarrollan estas actividades.Esta transición no sólo conduciría a un nuevo modo de producción sino a un modo de vida alternativo, a una nueva civilización; asimismo, y basado en el Objetivo $N^{0} 5$, plantea líneas de acción de importancia en la gestión ambiental, incluyendo a las ABRAE, las cuales desempeñan un importante rol en los aspectos de geopolítica, conservación de bienes naturales y como estrategia ante el cambio climático.

A partir del Primer Plan Nacional de la Patria Simón Bolívar (2006-2013) promulgado en el año 2006, en la República Bolivariana de Venezuela y puesta en marcha de sus directrices, se impulsó un nuevo modelo productivo nacional y una nueva geopolítica, tanto nacional como internacional, derivándose de allí la creación de nuevas unidades de producción para la satisfacción de las necesidades humanas en contraposición a la mera producción de riqueza.

Posteriormente, en el Plan Patria 2013-2019, se presentan los grandes objetivos históricos de la Revolución Bolivariana: La Independencia Nacional, El Socialismo Bolivariano, Venezuela País Potencia, El Mundo Pluricéntrico y Pluripolar y la 
Preservación de la Vida en el Planeta, donde se plasma la participación protagónica en la gestión corresponsable de gobierno y su integración a los planes de desarrollo con la visualización del Ecosocialismo, como elemento transversal a los cinco objetivos históricos.

En la esenciafilosófica del Ecosocialismo, se articulan entre sí elementos importantes como lo son el territorio, el ambiente y los procesos productivos. A través de esta visión se aspira transformar el modelo territorial y ambiental; de allí que el desarrollo es concebido como la puesta en marcha de unidades de producción donde se realice un impulso a la participación de los actores sociales en la construcción de las redes socioproductivas, partiendo de los bienes naturales disponibles, del territorio y de la racionalizada capacidad para asimilar los desechos generados, respetando las capacidades de los ecosistemas, y logrando una articulación que permita la construcción de procesos socioproductivos encadenadoscon las comunidades del territorio para elevar su calidad de vida, pero a la vez evitando y previniendo prácticas degradantes del ambiente. Por ello, la propuesta es una planificación participativa integral para elaborar los planes y proyectos en concordancia con los objetivos del plan de la Patria 2013-2019.

\section{CONCLUSIONES}

De acuerdo a la experiencia habida y a los nuevos lineamientos a seguir, se impone para la ordenación del territorio, una estrategia metodológica integradora de los aspectos naturales, físicos, socioeconómicos y culturales, donde se siga una línea de trabajo en la cual se considere cada espacio a ordenar, como parte de un sistema en constante reestructuración; donde los elementos naturales y sociales están en interacción, estableciéndose entre ellos relaciones simultáneas de dependencia y reciprocidad (MINAMB, 2007); establecer y mantener vínculos comunicacionales y de trabajo entre los actores sociales (individuos e instituciones) que interactúen dentro de cada área haciéndolos copartícipes en los instrumentos de gestión.

La metodología seguida en el MINAMB (hoy Ministerio de Ecosocialismo y Aguas), en los más recientes planes de ordenamiento territorial,por ser integradora, permitió formar un espiral de aprendizaje colectivo, de reconstrucción de la realidad y de sinergia y empatía por parte de los actores e implicó un replanteamiento epistemológico, político y metodológico; desde una óptica holística, con y para la comunidad, en concordancia con el entorno ambiental induciendo a tomar de él, solo lo necesario y a respetar los ciclos de producción de los ecosistemas. 
En general, la planificación participativa vinculada al ordenamiento territorial busca, desechar modelos y visiones que ya no resuelven los problemas de la gente, sino ubicarse en las tendencias que apuntan al desarrollo, armonizar los esfuerzos públicos y privados, darle viabilidad abriendo cauces a los emprendimientos en concordancia con el ambiente; realizándolo de forma interactuada con las comunidades en todas sus fases e implica reducir la brecha entre el marco legal preexistente y los nuevos instrumentos jurídicos, para conseguir la consonancia requerida entre lo que dice la ley en Venezuela, y lo que se aspira en el marco del Ecosocialismo.

\section{AGRADECIMIENTOS:}

Al Ministerio del Poder Popular para el Ecosocialismo y Aguas (MINEA), Dirección de Gestión Territorial por suministro de material de apoyo.

\section{BIBLIOGRAFÍA}

- Berroterán, José L. (2204). Reserva Forestal Imataca. Ecología y Bases Técnicas para el Ordenamiento Territorial. Fundación U.C.V. Caracas, Venezuela.

- CEPAL, (2001). El Ordenamiento Territorial como Opción de Políticas Urbanas y Regionales en América Latina y el Caribe. Documento presentado por Pedro Felipe Montes Lira. Serie: Medio Ambiente y Desarrollo; División de Medio Ambiente y Desarrollos Humanos. Santiago de Chile, Chile.

- García, R., (2014). Plan de Ordenación Urbano Local: Lineamientos para su elaboración. Informe Técnico. Instituto Geográfico de Venezuela Simón Bolívar, Dirección de Catastro. Caracas, Venezuela.

- González E., Roque, (1983). Planeación y Reglamentación del Desarrollo Urbano. Gaceta Mexicana de Administración Pública Estatal y Municipal. Ponencia presentada durante el Seminario para la Formación de Capacitadores Municipales, Guadalajara, Jalisco. 1983. México.

- Hernández, R., Fernández, C. y Baptista, L., (2010). Metodología de la Investigación. 5ta. Edición. McGraw-Hill Interamericana. México.

- Lowvy, M., (2004). Que es el Ecosocialismo. México, México. 
- Méndez M., (2013). Diario El Impulso. Artículo publicado en prensa e17.10.2013

- Ministerio del Poder Popular para el Ambiente, (2013). Dibujando nuestro Ecosocialismo. Resúmenes de Ponencias. IV Congreso Venezolano de Diversidad Biológica. Estado Falcón, Venezuela.

- Ministerio del Poder Popular Para el Ambiente, (2007). Experiencias de planificación participativa en la elaboración de los planes de ordenamiento y reglamento de uso años 2005-2007. (Estudio de casos). Documento Interno. Dirección General de Planificación y Ordenación Ambiental. Documento interno. Caracas Venezuela.

- Ministerio del Poder Popular para el Transporte y las Comunicaciones, (2011). Inventario de Instrumentos de Planificación Vigentes - Publicados. Dirección General de Ordenación del Territorio Urbanístico. Caracas, Venezuela.

- Ministerio del Poder Popular para el Ambiente (2008). Análisis Ecológico Social y Ordenamiento Territorial de la Región del Río Caura. Documento Técnico. DGPOA. Caracas, Venezuela.

- República Bolivariana de Venezuela, (2000). Constitución de la República Bolivariana de Venezuela. Gaceta Oficial Extraordinaria No 5.453. 24 de marzo de 2000. Caracas.

- República Bolivariana de Venezuela, (2000). Ley de Geografía, Cartografía y Catastro Nacional. Gaceta Oficial Extraordinaria No 37.002. 28 de julio de 2000. Caracas.

- República Bolivariana de Venezuela (2014). Ley Orgánica de Seguridad de la Nación. Gaceta Oficial Extraordinaria № 6.156, de fecha 19 de noviembre de 2014.

- República Bolivariana de Venezuela (2005). Ley Orgánica de Pueblos y

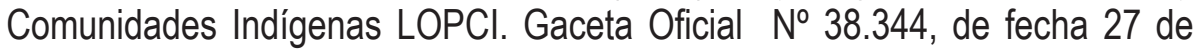
diciembre de 2005.

- República Bolivariana de Venezuela, (2006). Ley Orgánica del Ambiente. Gaceta Oficial Extraordinaria № 5.833, de fecha 22 de diciembre de 2006.

- República Bolivariana de Venezuela. (2009). Ley de Gestión Integral de Riesgos Socionaturales y Tecnológicos. Gaceta Oficial Extraordinaria № 39.095 del 9 de enero de 2009 
- República Bolivariana de Venezuela, (2008). Ley de Gestión de la Diversidad Biológica (2008). Gaceta Oficial 30.060 Fecha 01 de diciembre de 2008.

- República Bolivariana de Venezuela, (2009). Ley Orgánica de los Consejos Comunales (2009). Gaceta Oficial No 39.335. Fecha 28 de diciembre de 2009.

- República Bolivariana de Venezuela (2020). Ley de Planificación Pública y Popular. Gaceta Oficial-E. Nº 6-001. Fecha 21 de diciembre de 2010.

- República Bolivariana de Venezuela (2013). Ley del Plan de la Patria. Segundo Plan Socialista de Desarrollo Económico y Social de la Nación 2013-2019. Gaceta Oficial-E. Nº 6.118. Fecha 04 de diciembre de 2013.

- República Bolivariana de Venezuela (2014). Ley Orgánica de las Comunas y Movimientos Sociales. Gaceta Oficial Extraordinaria N ${ }^{0}$ 409.327, de fecha 07 de febrero de 2014.

- República de Venezuela, (1936). Ley de Tierras Baldías y Ejidos. Gaceta Oficial de Fecha 30 de septiembre de 1936.

- República de Venezuela, (1983). Ley Orgánica de Ordenación del Territorio. Gaceta Oficial No 3.238-Extraordinario, del 11 de agosto de 1983.

\section{Enlaces Web}

- http://www.monografias.com/trabajos14/segurid-nacion/segurid-nacion. shtml\#ixzz3aXblyvuu. (Consultado el 7/07/15

- https://es.scribd.com/.../Michael-Lowy-Ecosocialismo-La-alternativa-radical. Consultadoel 03/08/15

- http://www.hic-al.org/glosario_definicion.cfm?id_entrada=22

- SHOP/CEPAL/PNUMA, Ecotécnicas para los asentamientos humanos en el trópico húmedo de México, marzo de 1978. Consultado el 26/07/15)

- http://madalbo.com/2011/08/crisis-ecologica-y-lucha-politica-la.html.

- Crisis ecológica y lucha política: la alternativa ecosocialista.

- Michael Lowvy y Samuel González. Consultado el 03/08/15 